\title{
Shortening the Edinburgh postnatal depression scale using optimal test assembly methods: Development of the EPDS-Dep-5
}

\author{
Daphna Harel $^{1,2}$ (1) | Brooke Levis $^{3,4,5}$ | Miyabi Ishihara ${ }^{6}$ | Alexander W. Levis ${ }^{7}$ | Simone

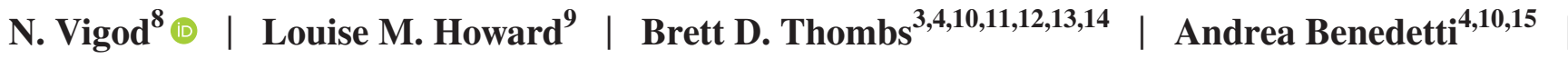 \\ the DEPRESsion Screening Data (DEPRESSD) EPDS Collaboration*
}

${ }^{1}$ PRIISM Applied Statistics Center, New York University, New York, NY, USA

${ }^{2}$ Department of Applied Statistics, Social Science, and Humanities, New York University, New York, NY, USA

${ }^{3}$ Lady Davis Institute for Medical Research, Jewish General Hospital, Montréal, QC, Canada

${ }^{4}$ Department of Epidemiology, Biostatistics and Occupational Health, McGill University, Montréal, QC, Canada

${ }^{5}$ Centre for Prognosis Research, School of Medicine, Keele University, Staffordshire, UK

${ }^{6}$ Department of Statistics, University of California Berkeley, Berkeley, California, USA

${ }^{7}$ Department of Biostatistics, Harvard T.H. Chan School of Public Health, Harvard University, Boston, MA, USA

${ }^{8}$ Women's College Hospital and Research Institute, University of Toronto, Toronto, ON, Canada

${ }^{9}$ Institute of Psychiatry, Psychology \& Neuroscience, King's College London, London, UK

${ }^{10}$ Department of Medicine, McGill University, Montréal, QC, Canada

${ }^{11}$ Department of Psychiatry, McGill University, Montréal, QC, Canada

${ }^{12}$ Department of Psychology, McGill University, Montréal, QC, Canada

${ }^{13}$ Department of Educational and Counselling Psychology, McGill University, Montréal, QC, Canada

${ }^{14}$ Biomedical Ethics Unit, McGill University, Montréal, QC, Canada

${ }^{15}$ Respiratory Epidemiology and Clinical Research Unit, McGill University Health Centre, Montréal, QC, Canada

\section{Correspondence}

Daphna Harel, PRIISM Applied Statistics Center, New York University, 246 Greene Street, 3rd floor, New York NY 10003, USA.

Email: daphna.harel@nyu.edu

\section{Funding information \\ This study was funded by the Canadian Institutes of Health Research (CIHR, KRS-140994). Dr. Levis was supported by a Fonds de recherche du Québec- Santé (FRQS) Postdoctoral Training Fellowship. Drs. Thombs and Benedetti were supported by FRQS researcher salary awards. Dr. Wu was supported by a FRQS Postdoctoral Training Fellowship. Mr. Bhandari was supported by a studentship from the Research Institute of the McGill University Health Centre. Ms. Neupane was supported by G.R.}

\begin{abstract}
Aims: This study used a large database to develop a reliable and valid shortened form of the Edinburgh Postnatal Depression Scale (EPDS), a self-report questionnaire used for depression screening in pregnancy and postpartum, based on objective criteria. Methods: Item responses from the 10-item EPDS were obtained from 5157 participants (765 major depression cases) from 22 primary screening accuracy studies that compared the EPDS to the Structured Clinical Interview for DSM (SCID). Unidimensionality of the EPDS latent construct was verified using confirmatory factor analysis, and an item response theory model was fit. Optimal test assembly (OTA) methods identified a maximally informative shortened form for each possible scale length between 1 and 9 items. The final shortened form was selected based on prespecified validity and reliability criteria and non-inferiority of screening accuracy of the EPDS as compared to the SCID.
\end{abstract}

**The DEPRESsion Screening Data (DEPRESSD) EPDS Collaboration Group Authors are listed in Appendix. 
Caverhill Fellowship from the Faculty of Medicine, McGill University. Ms. Rice was supported by a Vanier Canada Graduate Scholarship. Ms. Azar was supported by a FRQS Masters Training Award. The primary study by Barnes et al was supported by a grant from the Health Foundation (1665/608). The primary study by Beck et al was supported by the Patrick and Catherine Weldon Donaghue Medical Research Foundation and the University of Connecticut Research Foundation. The primary study by Helle et al was supported by the Werner Otto Foundation, the Kroschke Foundation, and the Feindt Foundation. The primary study by de Figueiredo et al was supported by Fundação de Amparo à Pesquisa do Estado de São Paulo. The primary study by Tendais et al was supported under the project POCI/SAU-ESP/56397/2004 by the Operational Program Science and Innovation 2010 (POCI 2010) of the Community Support Board III and by the European Community Fund FEDER. This primary study by Green et al was supported by a grant from the Duke Global Health Institute (4530751). The primary study by Kettunen et al was supported with an Annual EVO Financing (Special government subsidies from the Ministry of Health and Welfare, Finland) by North Karelia Central Hospital and Päijät-Häme Central Hospital. The primary study by Phillips et al was supported by a scholarship from the National Health and Medical and Research Council (NHMRC). The primary study by Nakić Radoš et al was supported by the Croatian Ministry of Science, Education, and Sports (1340000000-2421). The primary study by Rochat et al was supported by grants from the University of Oxford (HQ5035), the Tuixen Foundation (9940), the Wellcome Trust (082384/Z/07/Z and 071571), and the American Psychological Association. Dr. Rochat receives salary support from a Wellcome Trust Intermediate Fellowship (211374/Z/18/Z). The primary study by Smith-Nielsen et al was supported by a grant from the charitable foundation Tryg Foundation (Grant ID no 107616). The primary study by Prenoveau et al was supported by The Wellcome Trust (grant number 071571). The primary study by Stewart et al was supported by Professor Francis Creed's Journal of Psychosomatic Research Editorship fund (BA00457) administered through University of Manchester. The primary study by Tandon et al was funded by the
Results: A 5-item short form of the EPDS (EPDS-Dep-5) was selected. The EPDSDep-5 had a Cronbach's alpha of 0.82. Sensitivity and specificity of the EPDS-Dep-5 for a cutoff of 4 or greater were 0.83 (95\% CI, 0.73, 0.89) and 0.86 (95\% CI, 0.80 , $0.90)$ and were statistically non-inferior to the EPDS. The correlation of total scores with the full EPDS was high $(r=0.91)$.

Conclusion: The EPDS-Dep-5 is a valid short form with minimal loss of information when compared to the full-length EPDS. The EPDS-Dep-5 was developed with OTA methods using objective, pre-specified criteria, but the approach is data-driven and exploratory. Thus, there is a need to replicate results of this study in different populations.

\section{K E Y W O R D S}

depression, optimal test assembly, patient-reported outcome, short form 
Thomas Wilson Sanitarium. The primary study by Tran et al was supported by the Myer Foundation who funded the study under its Beyond Australia scheme. Dr. Tran was supported by an early career fellowship from the Australian National Health and Medical Research Council. The primary study by Vega-Dienstmaier et al was supported by Tejada Family Foundation, Inc, and Peruvian-American Endowment, Inc. No other authors reported funding for primary studies or for their work on the present study. No sponsor or funder was involved in the study design; in the collection, analysis and interpretation of the data; in the writing of the report; or in the decision to submit the paper for publication.

\section{1 | INTRODUCTION}

Depression is a leading cause of disability among women. ${ }^{1}$ Although the $7-13 \%$ prevalence of major depression during pregnancy and postpartum ${ }^{2-5}$ is similar to rates among women during non-childbearing periods, ${ }^{3,6-10}$ perinatal depression is associated with adverse outcomes for the mother, developing child, mother-infant relationship and marital quality. ${ }^{11-13}$ Most women with depression in the perinatal period, however, do not receive adequate care. ${ }^{14-16}$ Rapidly identifying women with depression to improve their care is a high clinical priority. ${ }^{17}$

The 10-item Edinburgh Postnatal Depression Scale (EPDS) is the most commonly used self-report questionnaire in pregnancy and postpartum for screening, and it is also used as a continuous scale for symptom monitoring clinically and for research. ${ }^{16,18}$ Scores on each EPDS item reflect the frequency of symptoms in the last two weeks and range from 0 to 3 , with questions 3 and 5-10 reverse coded. Total scores range from 0 to 30 . Higher scores indicate greater depressive symptomatology. As completing measures can be demanding, shortened versions with scores that perform comparably well with original full-length versions may help reduce the burden placed on respondents, as well as decrease the time it takes to administer the scale. However, shortening a scale is only advisable if it does not adversely affect measurement and screening accuracy properties of the scale.

Shortened forms of the full 10-item EPDS have been developed Table 1. ${ }^{19-24}$ These include two two-item forms, ${ }^{19,24}$ a five-item form, ${ }^{20}$ three- and seven-item subscales that measure symptoms of anxiety and depression separately, ${ }^{21,24}$ a three-item form, ${ }^{22}$ and an eight-item form. ${ }^{23}$ None of the development processes for these shortened forms used pre-specified criteria for performance to determine how many items to remove from the full 10-item

\section{Significant Outcomes}

- A 5-item short form of the EPDS can be used to screen for depression in the perinatal period.

- The 5-item short form was shown to be valid and reliable in a sample of 5157 participants.

- Optimal test assembly methods provide a replicable and reproducible methodology to shorten patient-reported outcomes.

\section{Limitations}

- This study was not able to obtain data from 25 of 81 eligible datasets.

- There exists substantial heterogeneity across studies in terms of country and language of administration of the semi-structured interview.

- The optimal test assembly procedure is datadriven and should be replicated.

EPDS. Furthermore, only three studies shortening the EPDS validated against major depression classification status, ${ }^{20,22,24}$ and these studies included only 63, 19, and 9 major depression cases. The extent to which the existing shortened forms retain the measurement and diagnostic properties of the full scale is unclear. Individual participant data meta-analysis (IPDMA), in which participant-level data from many studies are synthesized, allows for the development of a shortened form using data from a large number of participants.

Optimal test assembly (OTA) is a mixed-integer programming procedure that uses an estimated item response theory (IRT) model to select the subset of items that maximizes performance with respect to a given metric while satisfying pre-specified constraints. ${ }^{25}$ While more commonly 

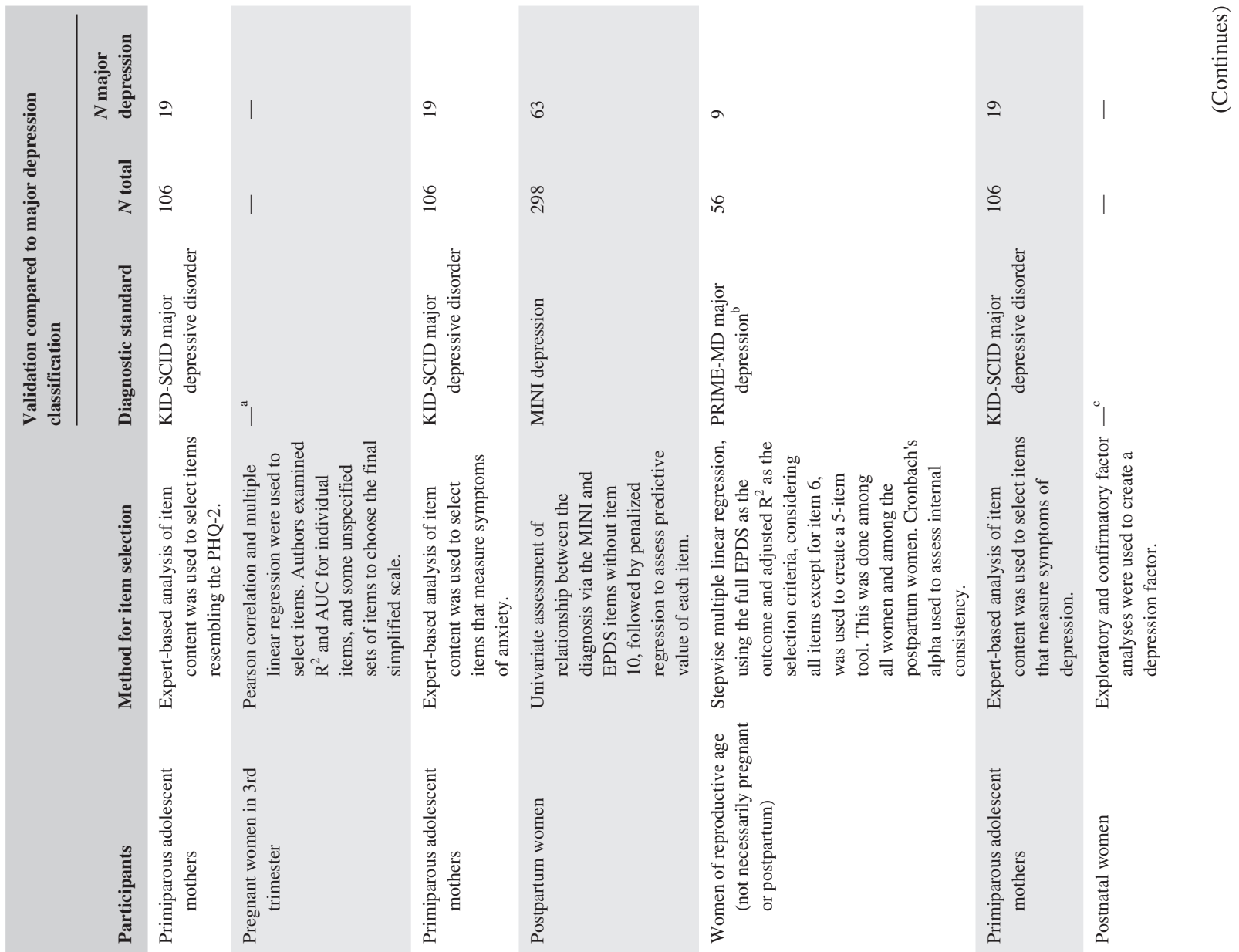

$\mid$ 彭

. 8

$\infty \mid$ 焉 $x \quad x$

$-\mid \frac{\text { के }}{\text { क }}$

के

हू.

है

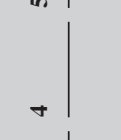

产

ल 产

N|

-1 音

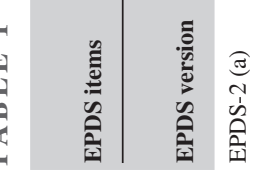

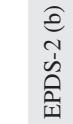

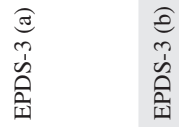

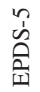

藏 空 


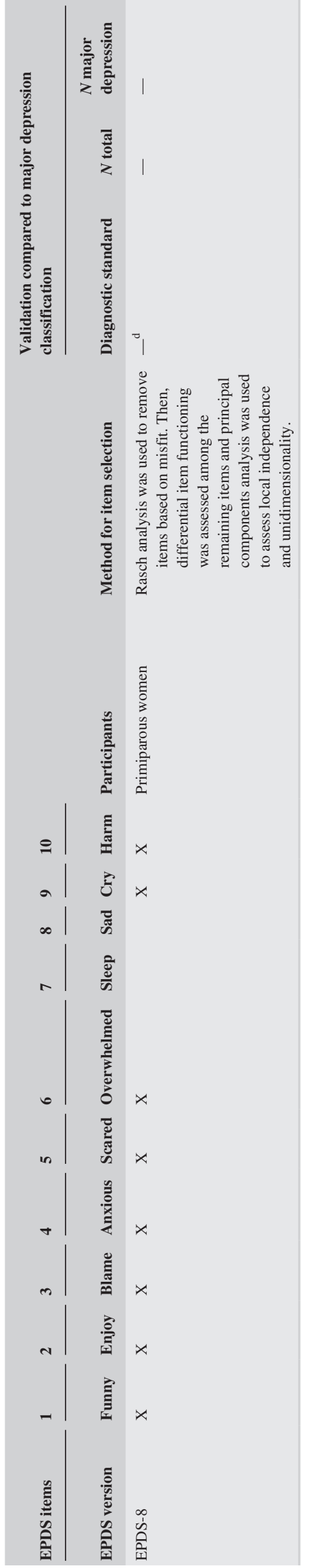

used in the development of high-stakes educational tests, ${ }^{26}$ OTA is being increasingly used to develop shortened versions of patient-reported outcome measures. ${ }^{27-29}$ This procedure was also shown to be replicable, reproducible, and to produce shortened forms of minimal length compared to alternative methods. ${ }^{30}$

\subsection{Aims of the study}

The objective of the present study was to apply optimal test assembly methods to a large database in order to develop a shortened version of the Edinburgh Postnatal Depression Scale. We (1) used confirmatory factor analysis to verify the unidimensionality of the underlying construct measured by the Edinburgh Postnatal Depression Scale; (2) applied optimal test assembly methods to obtain candidate forms of each possible length; and (3) selected the shortest possible form that showed similar performance to the full form in terms of pre-specified validity, reliability, and screening accuracy criteria, compared to the Edinburgh Postnatal Depression Scale.

\section{2 | MATERIALS AND METHODS}

This study used a subset of data accrued for an IPDMA on the diagnostic accuracy of the EPDS for screening to detect major depression among pregnant and postpartum women. This IPDMA was registered in PROSPERO (CRD42015024785) and a protocol was published. ${ }^{31}$ The protocol for the main IPDMA did not include methods for the present study. A protocol for the present study was uploaded to the Open Science Framework repository prior to initiating the study (https:// osf.io/3cepr/).

\subsection{Study eligibility for the main IPDMA}

Datasets from articles in any language were eligible if they included women $\geq 18$ years who were pregnant or had given birth in the previous year and both: (a) EPDS scores and (b) diagnostic classification for a current Major Depressive Episode (MDE) using Diagnostic and Statistical Manual of Mental Disorders (DSM) or International Classification of Diseases (ICD) criteria based on a validated semi-structured or fully structured interview, administered within two weeks of each other. Participants recruited from psychiatric settings or setting where scales or interviews were administrated because of reported symptoms of depression were excluded, since screening is done to identify previously unrecognized cases. ${ }^{32}$ Not all participants in a dataset needed to be eligible, if primary data allowed the selection of eligible participants. 


\subsection{Database searches and study selection}

A medical librarian searched Medline, Medline In-Process \& Other Non-Indexed Citations and PsycINFO via OvidSP, and Web of Science Core Collections via ISI Web of Knowledge from inception to October 3, 2018, using a peer-reviewed ${ }^{33}$ search strategy (Methods S1). We reviewed reference lists of relevant reviews and queried contributing authors about non-published studies. Search results were uploaded into RefWorks (RefWorks-COS). After de-duplication, remaining citations were uploaded into DistillerSR (Evidence Partners) for processing review results.

Two investigators independently reviewed titles and abstracts. If either deemed a study potentially eligible, full-text review was done by two investigators, independently, with disagreements resolved by consensus, consulting a third investigator when necessary.

\section{3 | Data contribution, extraction, and synthesis}

Authors of eligible datasets were invited to contribute deidentified primary data, including EPDS item scores and major depression status. We emailed corresponding authors of eligible primary studies at least three times, as necessary. If there was no response, we emailed co-authors and attempted phone contact.

Individual participant data were converted to a standard format and synthesized into a single dataset. We compared published participant characteristics and accuracy results with results from raw datasets and resolved any discrepancies in consultation with primary investigators.

For defining major depression, we considered MDD or MDE based on the DSM or ICD. If more than one was reported, we prioritized MDE over MDD. This is because screening would attempt to detect depressive episodes; further interview would determine if the episode is related to MDD, bipolar disorder, or persistent depressive disorder. We also prioritized DSM over ICD.

When datasets included statistical weights to reflect sampling procedures, we used the provided weights. For studies where sampling procedures merited weighting (e.g., all participants with positive screens and a random subset of participants with negative screens received a diagnostic interview), but the original study did not weight, we used inverse selection probabilities.

\section{4 | Data eligibility for present study}

For the present study, from the main IPDMA dataset, we only included primary studies that classified major depression based on the Structured Clinical Interview for DSM (SCID) ${ }^{34}$ The SCID is a semi-structured diagnostic interview that was designed to be conducted by experienced diagnosticians. It requires clinical judgment and allows rephrasing questions and probes to follow up responses. Fully structured interviews, on the other hand, are fully scripted, with no allowance for deviation from the script. These interviews remove clinical judgement from the process, allowing lay interviewers, rather than clinicians, to perform the assessment. Because of this, they may sacrifice validity. In recent analyses using three large IPDMA databases, ${ }^{35-37}$ it was found that compared to semi-structured interviews, fully structured interviews, which are designed for administration by lay interviewers, may identify more patients with low-level symptoms as depressed but fewer patients with high-level symptoms. Furthermore, a very brief version, the Mini International Neuropsychiatric Interview, identified far more participants as being depressed across the symptom spectrum. ${ }^{35-37}$ These results were consistent with the idea that semi-structured interviews most closely replicate clinical interviews done by trained professionals, whereas fully structured interviews are less rigorous reference standards. They are less resource-intensive options that can be administered by research staff without diagnostic skills but may misclassify major depression in substantial numbers of patients. Semi-structured interviews replicate diagnostic standards more closely than other types of interviews, and the SCID is by far the most commonly used semi-structured diagnostic interview for depression research [34-36]. In our main EPDS IPDMA database, 34 of 36 studies that used semi-structured interviews to classify major depression status used the SCID. Therefore, we only included SCID studies.

In addition, as EPDS item-level data was necessary for the proposed analyses, we only included studies in which EPDS item-level data (not just total scores) were available. For studies that collected data at multiple time points, we selected the time point with the most participants. If there was a tie, we selected the time point with the most major depression cases.

\subsection{Statistical analyses}

All analyses were conducted using $\mathrm{R}$ version 3.6.0.

\subsection{1 | Verification of unidimensionality of the EPDS}

Robust weighted least squares estimation in $\mathrm{R}$ was used to fit a single-factor confirmatory factor analysis model of EPDS items. ${ }^{38}$ The model was first fit without allowing 
for any residual correlations among the items. If there was poor model fit, and if warranted by theoretical justification, modification indices were to be used to identify item pairs that would improve model fit by allowing their residuals to correlate. ${ }^{39}$ Model fit was evaluated concurrently, using the $\chi^{2}$ statistic, Comparative Fit Index (CFI), Tucker-Lewis Index (TLI), and Root Mean Square Error of Approximation (RMSEA). ${ }^{40}$ Priority was given to CFI, TLI, and RMSEA, because the $\chi^{2}$ test may reject well-fitting models when sample size is large. ${ }^{41}$ Model fit was considered to be adequate if CFI and TLI were $\geq 0.95$ and RMSEA $\leq 0.08$. ${ }^{42}$ The confirmatory factor analysis was fit using the lavaan package. $^{43}$

\subsubsection{Item response theory model and optimal test assembly}

A generalized partial credit model (GPCM) was fit to EPDS pooling data from all included studies. ${ }^{44}$ The GPCM is an IRT model that relates a latent trait, representing severity of depressive symptomatology, to the distribution of observed item-level responses. The GPCM estimates two types of item-specific parameters: a discrimination parameter and threshold parameters. From these item-level parameter estimates, item information functions for each item were calculated from the GPCM, as well as a test information function (TIF), obtained by summing item information functions. Because the TIF is inversely related to the standard error of measurement of the latent trait, high amounts of information represent greater precision for measuring depressive symptomatology. The GPCM was fit using the ltm package. ${ }^{45}$

Next, we used OTA - a mixed-integer programming technique-to systematically search for the short form that maximized the TIF, subject to the constraint of fixing the number of items included in each short form. By using the TIF as the objective function, the procedure optimizes the precision of the short form in estimating participants' level of depressive symptomatology. ${ }^{25,46}$ The shape of the TIF was anchored at five points. ${ }^{25}$ Thus, for each short form of lengths 1-9 items, OTA selected items from the full set of EPDS items that maximized the test information. The OTA analysis was conducted using the lpSolveAPI package.

For each of the 9 candidate short forms and the full-length form, two scoring procedures were used to obtain estimates of each participant's level of depressive symptomatology. First, the summed scores across all items included in the short form were calculated. Second, factor scores were estimated for each participant. Although summed scores are typically relied upon for clinical use, the factor scores are considered to provide a better estimate of the latent trait because of well-known limitations of the summed score under the GPCM. ${ }^{4,48}$

\subsubsection{Selection of final short form}

The elimination of items necessarily reduces information compared to a full-length form. Thus, to guarantee adequate performance, the selection of the final short form was based on the following five criteria: reliability, concurrent validity of summed scores, concurrent validity of factor scores, and non-inferior sensitivity and specificity.

Reliability of each candidate short form was assessed with Cronbach's alpha, ${ }^{49}$ since it is commonly used in research, despite limitations. The final selected form was required a priori to have a Cronbach's alpha coefficient $\geq 0.80$. Concurrent validity of the summed scores and factor scores was measured with the Pearson's correlation coefficient between candidate

T A B L E 2 Patient demographic and diagnostic characteristics $(N=5157)$

\begin{tabular}{|c|c|}
\hline Sociodemographic variables & Summary \\
\hline Age, years, mean $[$ median] \pm SD (range) & $\begin{array}{c}29.1[29] \pm 5.9 \\
(18,47)\end{array}$ \\
\hline EPDS-10 score, mean [median] \pm SD (range) & $\begin{array}{c}7.1[6] \pm 5.9(0 \\
30)\end{array}$ \\
\hline \multicolumn{2}{|l|}{ Country, $n(\%)$} \\
\hline Australia & $158(3.1)$ \\
\hline Brazil & $241(4.7)$ \\
\hline Croatia & $272(5.3)$ \\
\hline Denmark & $320(6.2)$ \\
\hline Finland & $134(2.6)$ \\
\hline Germany & $224(4.3)$ \\
\hline Greece & $81(1.6)$ \\
\hline Hungary & $484(9.4)$ \\
\hline Italy & $29(0.6)$ \\
\hline Kenya & $161(3.1)$ \\
\hline Malawi & $186(3.6)$ \\
\hline Peru & $306(5.9)$ \\
\hline Portugal & $141(2.7)$ \\
\hline South Africa & $104(2.0)$ \\
\hline Thailand & $625(12.1)$ \\
\hline United Kingdom & $1093(21.2)$ \\
\hline United States of America & $239(4.6)$ \\
\hline Vietnam & $359(7.0)$ \\
\hline \multicolumn{2}{|l|}{ Pregnancy status, $n(\%)$} \\
\hline Pregnant & $1455(28.2)$ \\
\hline Postpartum & $3702(71.8)$ \\
\hline \multicolumn{2}{|l|}{ Classification system, $n(\%)$} \\
\hline DSM-III-R & $428(8.3)$ \\
\hline DSM-IV & $3947(76.5)$ \\
\hline DSM-IV-TR & $301(5.8)$ \\
\hline DSM-V & $481(9.3)$ \\
\hline
\end{tabular}


short form scores and the full-length EPDS. It was required a priori to be $\geq 0.90 .^{30}$

Diagnostic accuracy of each candidate short form was assessed through a three-step process. First, pooled sensitivity and specificity of each candidate short form (compared to the SCID) for each of its possible cutoff summed score values were estimated with a bivariate random-effects model. Second, for each candidate short form, an optimal cutoff score was selected using Youden's J statistic (sensitivity + specificity -1$){ }^{50,51}$ The bivariate random-effects model was fit using the lme4 package. ${ }^{52}$

Third, two non-inferiority tests were conducted for each of the 9 candidate forms to compare sensitivity and specificity, separately, to the full-length form. Non-inferiority tests assess whether the sensitivity or specificity of the short form is not lower than that of the full-length form, up to a pre-specified clinically significant tolerance of $\delta=0.05 .^{53}$ To conduct the non-inferiority test, the sampling distribution of the test statistic was generated through the bootstrap method. ${ }^{54}$
Bootstrapping resamples the original dataset with replacement to generate new, artificial, datasets. ${ }^{55}$ For each non-inferiority test, 2000 bootstrap iterations were conducted, controlling in each for the number of respondents with and without major depression. For each bootstrap iteration, the bivariate random-effects model was fit to each of the 9 candidate short forms and the full-length form, and the sensitivities and specificities were computed based on their cutoff scores. To account for the multiple testing in the 18 total non-inferiority tests, Benjamini-Hochberg adjusted $p$-values were used to determine the significance of the tests at the 0.05 significance level. ${ }^{56}$

\subsection{Funding and ethics}

The study sponsors had no role in study design; in the collection, analysis, and interpretation of data; in the writing of the report; or in the decision to submit the paper for publication.

\begin{tabular}{|lll|} 
Item number & Description & $\begin{array}{c}\text { Discrimination } \\
\text { parameter }\end{array}$ \\
\hline 1 & $\begin{array}{c}\text { I have been able to laugh and see the funny side of } \\
\text { things }\end{array}$ & 1.69 \\
\hline 2 & I have looked forward with enjoyment to things & 1.51 \\
\hline 3 & I have blamed myself unnecessarily when things & 1.04 \\
\hline 4 & $\quad$ went wrong & 1.13 \\
\hline 5 & I have been anxious or worried for no good reason & 1.16 \\
\hline 6 & I have felt scared or panicky for no very good reason & 1.11 \\
\hline 7 & Things have been getting on top of me & 1.39 \\
\hline 8 & I have been so unhappy that I have had difficulty & \\
\hline 9 & sleeping & 3.00 \\
\hline 10 & I have felt sad or miserable & 2.57 \\
\hline
\end{tabular}

TA B LE 3 EPDS items and discrimination parameters from the generalized partial credit model
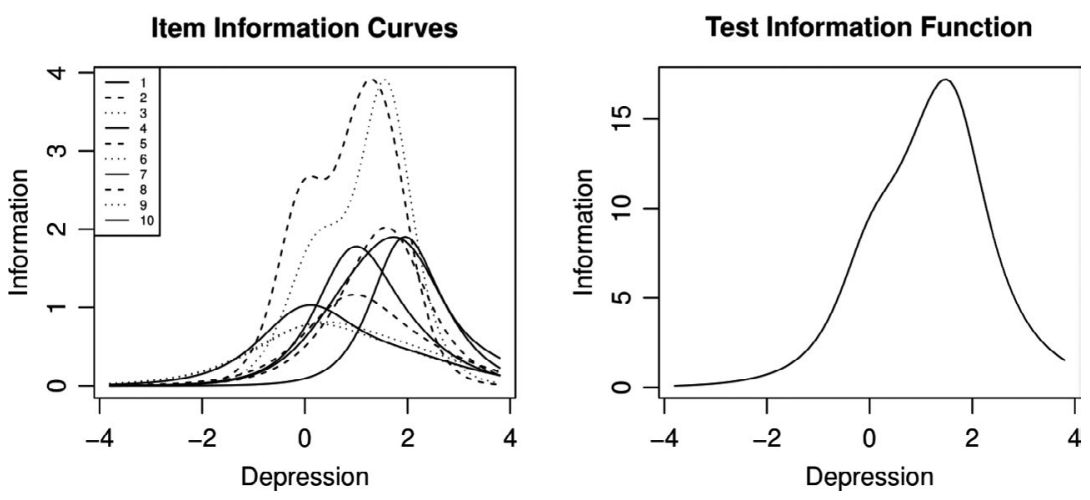

F I G U RE 1 The left-hand plot shows the item information functions for each of the 10 items. The right-hand plot shows the test information function of the EPDS 


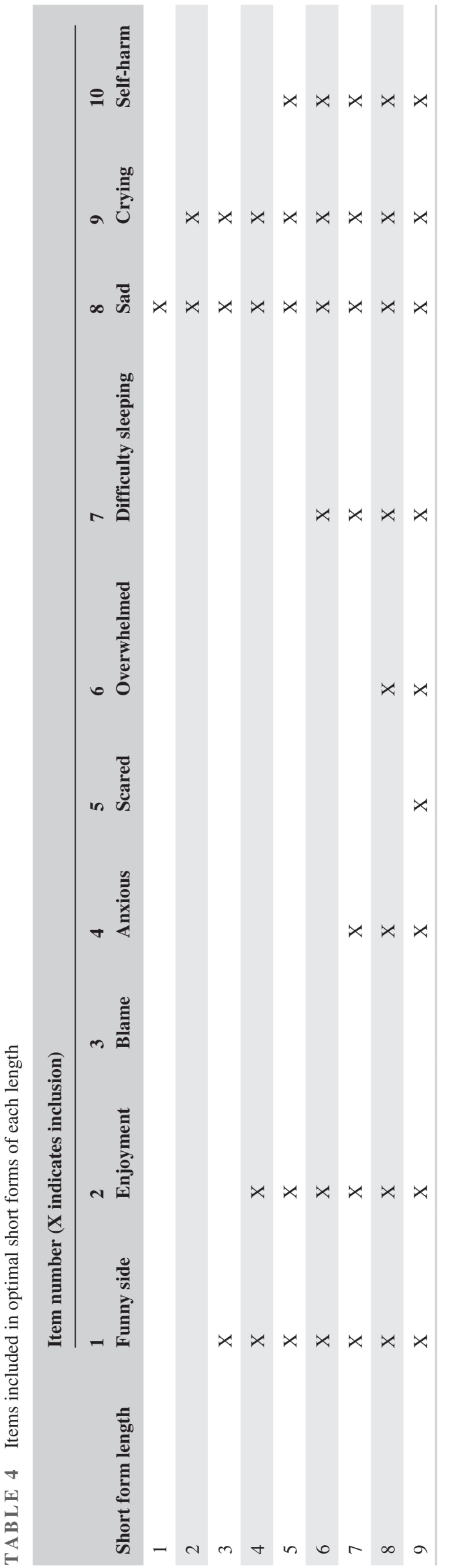

DH had full access to all data in the study and had final responsibility for the decision to submit for publication. As this study involved secondary analysis of de-identified previously collected data, the Research Ethics Committee of the Jewish General Hospital declared that this project did not require research ethics approval. However, for each included dataset, we confirmed that the original study received ethics approval and that all patients provided informed consent.

\section{3 | RESULTS}

\section{1 | Search results and inclusion of primary data}

Of 4434 unique titles and abstracts identified from the database search, 4056 were excluded after title and abstract review and 257 after full-text review, leaving 121 eligible articles with data from 81 unique participant samples, of which $56(69 \%)$ contributed datasets (Figure S1. Authors of included studies contributed data from two additional studies that were not retrieved by the search, for a total of 58 datasets. Of these, we excluded 24 studies that used a diagnostic interview other than the SCID and 12 more studies that did not have EPDS item scores available. In total, 5157 participants (765 major depression cases) from 22 primary studies were included. These studies were conducted in 18 different countries, with 17 different languages. The mean age of the sample was 29.1 years. See Table 2 for descriptive sample statistics and Table S1 for characteristics of each included study.

\section{2 | Unidimensionality of the EPDS}

A single-factor model was fit to the EPDS-10 with residuals modeled as uncorrelated $\left(\chi^{2}[\mathrm{df}=65]=663.1, p<0.0001\right.$, $\mathrm{TLI}=0.992, \mathrm{CFI}=0.988$, RMSEA $=0.042$ ). As this model was deemed to be well fitting, no modification indices were used. Factor loadings for items were all high, with a median of 0.97 and a range of 0.88 to 1.15 .

\section{3 | Item response theory model and optimal test assembly}

The discrimination parameters for each item based on the GPCM are presented in Table 3. The information functions of each of the 10 items, as well as the total TIF are shown in Figure 1. The item with the greatest discrimination parameter was item 8 , and thus has the most peaked information function in Figure 1. Other items with high values of the discrimination parameter and peaked information functions were 


\begin{tabular}{|c|c|c|c|}
\hline Form length & $\begin{array}{l}\text { Cronbach's } \\
\text { alpha }(95 \% \mathrm{CI})\end{array}$ & $\begin{array}{l}\text { Correlation of summed } \\
\text { scores }(95 \% \text { CI })\end{array}$ & $\begin{array}{l}\text { Correlation of factor } \\
\text { scores }(95 \% \mathrm{CI})\end{array}$ \\
\hline 1 & NA & $0.816(0.807,0.825)$ & NA \\
\hline 2 & $\begin{array}{l}0.811(0.797 \\
0.823)\end{array}$ & $0.867(0.860,0.874)$ & $0.913(0.841,0.953)$ \\
\hline 3 & $\begin{array}{l}0.805(0.794 \\
0.815)\end{array}$ & $0.891(0.885,0.897)$ & $0.932(0.875,0.963)$ \\
\hline 4 & $\begin{array}{l}0.833(0.823 \\
0.841)\end{array}$ & $0.899(0.894,0.904)$ & $0.945(0.898,0.970)$ \\
\hline 5 & $\begin{array}{l}0.818(0.808 \\
0.827)\end{array}$ & $0.910(0.906,0.915)$ & $0.949(0.906,0.973)$ \\
\hline 6 & $\begin{array}{l}0.840(0.832 \\
0.848)\end{array}$ & $0.932(0.928,0.935)$ & $0.962(0.930,0.980)$ \\
\hline 7 & $\begin{array}{l}0.844(0.837 \\
0.851)\end{array}$ & $0.965(0.963,0.967)$ & $0.975(0.953,0.987)$ \\
\hline 8 & $\begin{array}{l}0.856(0.850 \\
0.862)\end{array}$ & $0.980(0.979,0.981)$ & $0.988(0.977,0.993)$ \\
\hline 9 & $\begin{array}{l}0.868(0.862 \\
0.874)\end{array}$ & $0.991(0.990,0.991)$ & $0.997(0.993,0.998)$ \\
\hline 10 & $\begin{array}{l}0.877(0.872, \\
0.883)\end{array}$ & $1.000(1.000,1.000)$ & $1.000(1.000,1.000)$ \\
\hline
\end{tabular}

Note: Bold values represent those of the final selected form.

\begin{tabular}{|c|c|c|c|c|c|}
\hline Form length & $\begin{array}{l}\text { Optimal } \\
\text { cutoff }\end{array}$ & $\begin{array}{l}\text { Sensitivity } \\
(95 \% \mathrm{CI})\end{array}$ & $p$-value & $\begin{array}{l}\text { Specificity } \\
(95 \% \text { CI })\end{array}$ & $p$-value \\
\hline 1 & 2 & $\begin{array}{l}0.612(0.525 \\
0.693)\end{array}$ & 1.000 & $\begin{array}{l}0.917(0.884, \\
0.941)\end{array}$ & 0.000 \\
\hline 2 & 3 & $\begin{array}{l}0.654(0.563 \\
0.735)\end{array}$ & 1.000 & $\begin{array}{l}0.919(0.887 \\
0.943)\end{array}$ & 0.000 \\
\hline 3 & 3 & $\begin{array}{l}0.801(0.707 \\
0.870)\end{array}$ & 0.001 & $\begin{array}{l}0.823(0.762 \\
0.871)\end{array}$ & 0.822 \\
\hline 4 & 4 & $\begin{array}{l}0.782(0.670 \\
0.863)\end{array}$ & 0.022 & $\begin{array}{l}0.872(0.819 \\
0.912)\end{array}$ & 0.000 \\
\hline 5 & 4 & $\begin{array}{l}0.825(0.731, \\
0.892)\end{array}$ & 0.000 & $\begin{array}{l}0.859(0.801 \\
0.902)\end{array}$ & 0.000 \\
\hline 6 & 5 & $\begin{array}{l}0.803(0.720 \\
0.866)\end{array}$ & 0.000 & $\begin{array}{l}0.870(0.811, \\
0.913)\end{array}$ & 0.000 \\
\hline 7 & 7 & $\begin{array}{l}0.799(0.705 \\
0.869)\end{array}$ & 0.005 & $\begin{array}{l}0.892(0.833, \\
0.931)\end{array}$ & 0.000 \\
\hline 8 & 8 & $\begin{array}{l}0.834(0.751 \\
0.893)\end{array}$ & 0.000 & $\begin{array}{l}0.863(0.799 \\
0.909)\end{array}$ & 0.000 \\
\hline 9 & 9 & $\begin{array}{l}0.822(0.739 \\
0.882)\end{array}$ & 0.000 & $\begin{array}{l}0.857 \text { (0.793, } \\
0.904)\end{array}$ & 0.000 \\
\hline 10 & 11 & $\begin{array}{l}0.797(0.710 \\
0.863)\end{array}$ & NA & $\begin{array}{l}0.880(0.826 \\
0.919)\end{array}$ & NA \\
\hline
\end{tabular}

Note: Bold values represent those of the final selected form.

items 1, 2 and 9. Table 4 shows the items that were included in each of the 9 candidate short forms from the OTA analysis. Item 8 was included in all candidate short forms, with items 3,5 , and 6 quickly dropped.
TA B LE 5 Reliability and validity results of the candidate short forms

TA B LE 6 Screening accuracy results of the candidate short forms and their noninferiority test results

\subsection{Selection of final short form}

Cronbach's alpha values and concurrent validity correlations for the 9 candidate short forms are presented in Table 5. The 
results of the non-inferiority tests for both sensitivity and specificity are presented in Table 6 .

The 5-item short form (EPDS-Dep-5) was the shortest form that fulfilled all criteria. The form included item 1 ("I have been able to laugh and see the funny side of things"), item 2 ("I have looked forward with enjoyment to things"), item 8 ("I have felt sad or miserable"), item 9 ("I have been so unhappy that I have been crying"), and item 10 ("The thought of harming myself has occurred to me"). The EPDS-Dep-5 maintained high reliability with a Cronbach's alpha of 0.82 (95\% CI, 0.81, 0.83) compared to 0.88 (95\% CI, 0.87, 0.88) for the full-length form. Correlations of the summed and factor scores between the EPDS-Dep-5 and EPDS-10 were 0.91 (95\% CI, 0.91, 0.92) and 0.95 (95\% CI, 0.91, 0.97), respectively. Youden's J for the full EPDS and EPDS-Dep-5, at their optimal cutoffs of 11 or greater and 4 or greater, respectively, were both 0.68. Receiver operating curves for the full EPDS and EPDS-Dep-5 are presented in Figure S2. The sensitivity and specificity of the EPDS-Dep-5 at its optimal cutoff of 4 or greater were $0.83(95 \% \mathrm{CI}, 0.73,0.89)$ and $0.86(95 \%$ CI, 0.80, 0.90), respectively. Both sensitivity and specificity were non-inferior to the sensitivity $(0.80 ; 95 \% \mathrm{CI}, 0.71,0.86)$ and specificity $(0.88 ; 95 \% \mathrm{CI}, 0.83,0.92)$ of the full-length form.

\section{4 | DISCUSSION}

This study used OTA to shorten the EPDS to a 5-item shortened version (EPDS-Dep-5) while maintaining comparable measurement properties and screening accuracy to detect major depression among women in pregnancy and postpartum. The implication of this research is that shortening this scale allows for shorter administration times and places lower burden on respondents without significantly reducing the ability of the scale to measure depressive symptomology.

The EPDS-Dep-5 maintained similar sensitivity and specificity to that of the full-length form and resulted in a minimal loss of information. Furthermore, the shortened form maintained reliability and validity that were comparable to the full-length form based on pre-specified criteria. Cronbach's alpha of the EPDS-Dep-5 was within 0.06 of that for the full-length form, and correlations of the summed score and factor scores of the EPDS-5 and EPDS-10 were 0.91 and 0.95 . Per pre-specified criteria, the sensitivity and specificity of the EPDS-Dep-5 (0.825 and 0.859, respectively) were non-inferior to those of the EPDS-10 (0.797 and 0.880 , respectively).

The 5 items included in the EPDS-Dep- 5 included items $1,2,8,9$, and 10 from the original EPDS. These items cover the two core symptoms of depression-low mood (items 8 and 9) and anhedonia (items 1 and 2), as well as self-harm (item 10). Of note, although they were included as potential items for the final shortened form, none of the 3 anxiety items (items 3 [blame], 4 [anxious], and 5 [scared]) were retained in the EPDS-Dep-5. Our short form selection procedure assessed screening accuracy for detecting depression, not anxiety, and short form development for that purpose would need to be done separately.

Most existing studies developing shortened EPDS forms compared the shortened forms to the full EPDS rather than comparing to diagnostic classification for depression. Only three studies validated their shortened forms against major depression classification based on DSM or ICD diagnostic criteria, but these studies included only 63,19 , and 9 major depression cases, ${ }^{20,22,24}$ limiting their ability to draw conclusions about the shortened scales' measurement properties. Table 1 presents the items included in each study's shortened form as well as the methods used to create that version. The development of the EPDS-Dep-5 in the present study used data that originated from an IPDMA thus (1) providing the largest total sample size (5157 participants), as well as data from multiple settings and countries, (2) used by far the largest number of major depression cases (765 cases), (3) used a validated semi-structured diagnostic interview as the reference standard for major depression classification (the SCID), and (4) used screening accuracy as part of the development process, not solely as a tool for validation. It was also the only study that used objective, pre-specified criteria for empirical selection of items to include in the short form.

This study showed that an EPDS-Dep-5 cutoff $\geq 4$ maximized combined sensitivity and specificity using Youden's $\mathrm{J} .{ }^{51}$ However, clinicians and researchers may consider use of a higher cutoff if their goal is to only capture patients with high depressive symptom levels or a lower cutoff if their goal is to avoid false negatives.

There are several limitations for this study that must be considered. First, for the collection of data for the full IPDMA, it was not possible to obtain primary data from 25 of the 81 eligible datasets. In addition, of the 34 studies using the SCID that provided data for the full IPDMA, 12 did not provide EPDS item scores and thus could not be included in the present study. Second, although we included data from 22 studies that fulfilled strict inclusion criteria, including the use of the rigorous semi-structured SCID interview, there was still substantial heterogeneity across studies in terms of country and language which both allows for the generalization of the results to larger and more diverse populations but also may not select the optimal shortened form for each individual context. Third, the present study did not conduct a risk of bias assessment; however, the full IPDMA from which a subset of data was selected for this study did conduct a risk of bias assessment using QUADAS-2. No QUADAS-2 domain items were consistently associated 
with differences in sensitivity or specificity estimates. Furthermore, the OTA procedure, is a data-driven approach, and therefore the results of this study should be replicated or cross-validated. Lastly, future work may consider assessing whether the EPDS-Dep-5 is subject to issues of poor item fit or differential item functioning.

\section{5 | CONCLUSION}

The study used the OTA method to develop a valid and reliable 5-item shortened form of the EPDS using pre-specified objective criteria to determine the length and items included in the EPDS-Dep-5. This method was implemented with a sample of 5157 participants from 22 primary studies. The resulting 5-item shortened version maintained measurement properties and screening accuracy of the full-length form within pre-specified limits.

\section{CONFLICT OF INTEREST}

All authors have completed the ICJME uniform disclosure form and declare: no support from any organization for the submitted work; no financial relationships with any organizations that might have an interest in the submitted work in the previous three years with the following exceptions: Dr. Vigod declares that she receives royalties from UpToDate, outside the submitted work. Dr. Tonelli declares that he has received a grant from Merck Canada, outside the submitted work. Dr. Beck declares that she receives royalties for her Postpartum Depression Screening Scale published by Western Psychological Services. All authors declare no other relationships or activities that could appear to have influenced the submitted work. No funder had any role in the design and conduct of the study; collection, management, analysis, and interpretation of the data; preparation, review, or approval of the manuscript; and decision to submit the manuscript for publication.

\section{AUTHOR CONTRIBUTIONS}

DH, BL, SNV, BDT, AB, JTB, PC, SG, JPAI, LAK, SBP, IS, RCZ, LC, NDM and MTonelli were responsible for the study conception and design. JTB and LAK designed and conducted database searches to identify eligible studies. JB, CTB, CB, FPdF, GF, BF, EPG, NH, PAK, JK, ZK, AAL, SNR, TJR, JSN, AS, RCS, MTadinac, SDT, IT, AT, TDT, KT, MSV and JMVD contributed primary datasets that were included in this study. BL, YS, CH, AK, YW, PMB, DN, ZN, MImran, DBR, MA, MJC, NS, KER and BDT contributed to data extraction and coding for the individual participant data meta-analysis. DH, MIshihara and AWL conducted analyses and interpreted results. $\mathrm{DH}$ and $\mathrm{BL}$ drafted the manuscript. All authors provided a critical review and approved the final manuscript. DH is the guarantor. BDT and AB have full access to all the data in the study and take responsibility for the integrity of the data and the accuracy of the data analyses.

\section{PEER REVIEW}

The peer review history for this article is available at https:// publons.com/publon/10.1111/acps.13272.

\section{DATA AVAILABILITY STATEMENT}

Requests for data access should be made to Dr. Brooke Levis (brooke.levis@gmail.com).

\section{ORCID}

Daphna Harel (D) https://orcid.org/0000-0001-7015-5989

Simone N. Vigod (D) https://orcid.org/0000-0002-2736-9639

\section{REFERENCES}

1. Kessler RC. Epidemiology of women and depression. J Affect Disord. 2003;74(1):5-13. https://doi.org/10.1016/s0165-0327 (02)00426-3

2. Bennett HA, Einarson A, Taddio A, Koren G, Einarson TR. Prevalence of depression during pregnancy: systematic review. Obstet Gynecol. 2004;103(4):698-709. https://doi.org/10.1097/01 AOG.0000116689.75396.5f

3. Gavin NI, Gaynes BN, Lohr KN, Meltzer-Brody S, Gartlehner G Swinson T. Perinatal depression - a systematic review of prevalence and incidence. Obstetr Gynecol. 2005;106(5):1071-1083. https://doi.org/10.1097/01.AOG.0000183597.31630.db

4. Gaynes BN, Gavin N, Meltzer-Brody S, et al. Perinatal Depression: Prevalence, Screening Accuracy, and Screening Outcomes: Evidence Report/Technology Assessment, Number 119. Rockville, MD: Agency for Healthcare Research and Quality (US).

5. O'hara MW, Swain AM. Rates and risk of postpartum depression - a meta-analysis. Int Rev Psychiatry. 1996;8(1):37-54. https://doi org/10.3109/09540269609037816

6. Cooper PJ, Campbell EA, Day A, Kennerley H, Bond A. Nonpsychotic psychiatric-disorder after childbirth - a prospective-study of prevalence, incidence, course and nature. $\mathrm{Br} \mathrm{J}$ Psychiatry. 1988;152:799-806. https://doi.org/10.1192/bjp.152.6.799

7. Cox JL, Murray D, Chapman G. A controlled-study of the onset, duration and prevalence of postnatal depression. Br J Psychiatry. 1993;163:27-31. https://doi.org/10.1192/bjp.163.1.27

8. Ohara MW, Zekoski EM, Philipps LH, Wright EJ. Controlled prospective-study of postpartum mood disorders - comparison of childbearing and nonchildbearing women. J Abnorm Psychol. 1990;99(1):3-15. https://doi.org/10.1037/0021-843x.99.1.3

9. Stewart DE. Depression during pregnancy. N Engl J Med. 2011;365(17):1605-1611. https://doi.org/10.1056/NEJMcp1102730

10. Vesga-Lopez O, Blanco C, Keyes K, Olfson M, Grant BF, Hasin DS. Psychiatric disorders in pregnant and postpartum women in the United States. Arch Gen Psychiatry. 2008;65(7):805-815 https://doi.org/10.1001/archpsyc.65.7.805

11. Whitley R, Kirmayer LJ. Perceived stigmatisation of young mothers: an exploratory study of psychological and social experience. Soc Sci Med. 2008;66(2):339-348. https://doi.org/10.1016/j.socsc imed.2007.09.014 
12. Zelkowitz P, Milet TH. Postpartum psychiatric disorders: their relationship to psychological adjustment and marital satisfaction in the spouses. J Abnorm Psychol. 1996;105(2):281-285. https://doi org/10.1037/0021-843x.105.2.281

13. Zelkowitz P, Milet TH. The course of postpartum psychiatric disorders in women and their partners. J Nerv Ment Dis. 2001;189(9):575-582. https://doi.org/10.1097/00005053-20010 9000-00002

14. Duhoux A, Fournier L, Gauvin L, Roberge P. What is the association between quality of treatment for depression and patient outcomes? A cohort study of adults consulting in primary care. J Affect Disord. 2013;151(1):265-274. https://doi.org/10.1016/j. jad.2013.05.097

15. Duhoux A, Fournier L, Nguyen CT, Roberge P, Beveridge R. Guideline concordance of treatment for depressive disorders in Canada. Soc Psychiatry Psychiatr Epidemiol. 2009;44(5):385-392. https://doi.org/10.1007/s00127-008-0444-8

16. Howard LM, Molyneaux E, Dennis CL, Rochat T, Stein A, Milgrom J. Perinatal mental health 1 Non-psychotic mental disorders in the perinatal period. Lancet. 2014;384(9956):1775-1788 https://doi.org/10.1016/S0140-6736(14)61276-9

17. Canada MHCo. Changing Directions, Changing Lives: The Mental Health Strategy for Canada. Calgary, AB: Mental Health Commission of Canada; 2012.

18. HISCF, A. (2009). Alberta Postpartum Depression-Data Set. https://open.alberta.ca/dataset/0764f768-cd1a-4a70-88d4-afea1 c1b3a4d/resource/6da02b61-b9a4-4324-acdd-54465294a395/ download/perinatal-postpartum-depression-data-standard-versi on-1-0-2009-09-29.pdf.

19. Choi SK, Kim JJ, Park YG, Ko HS, Park IY, Shin JC. The Simplified Edinburgh Postnatal Depression Scale (EPDS) for antenatal depression: is it a valid measure for pre-screening? Int $\mathrm{J}$ Med Sci. 2012;9(1):40-46. https://doi.org/10.7150/ijms.9.40

20. Eberhard-Gran M, Eskild A, Samuelsen SO, Tambs K. A short matrix-version of the Edinburgh Depression Scale. Acta Psychiatr Scand. 2007;116(3):195-200. https://doi. org/10.1111/j.1600-0447.2006.00934.x

21. Gollan JK, Wisniewski SR, Luther JF, et al. Generating an efficient version of the Edinburgh Postnatal Depression Scale in an urban obstetrical population. J Affect Disord. 2017;208:615-620. https:// doi.org/10.1016/j.jad.2016.10.013

22. Martinez P, Magana I, Vohringer PA, Guajardo V, Rojas G. Development and validation of a three-item version of the Edinburgh Postnatal Depression Scale. J Clin Psychol. 2020;76(12):2198-2211. https://doi.org/10.1002/jclp.23041

23. Pallant JF, Miller RL, Tennant A. Evaluation of the Edinburgh Post Natal Depression Scale using Rasch analysis. BMC Psychiatry. 2006;6:Artn 28. https://doi.org/10.1186/1471-244x-6-28

24. Venkatesh KK, Zlotnick C, Triche EW, Ware C, Phipps MG. Accuracy of brief screening tools for identifying postpartum depression among adolescent mothers. Pediatrics. 2014;133(1):E45E53. https://doi.org/10.1542/peds.2013-1628

25. Linden WJ. Linear Models of Optimal Test Design. New York, NY: Springer; 2005.

26. Kuhn JT, Kiefer T. Optimal test assembly in practice the design of the Austrian educational standards assessment in mathematics. Z Psychol. 2013;221(3):190-200. https://doi. org/10.1027/2151-2604/a000146

27. Harel D, Mills SD, Kwakkenbos L, et al. Shortening patient-reported outcome measures through optimal test assembly: application to the Social Appearance Anxiety Scale in the Scleroderma Patient-centered Intervention Network Cohort. BMJ Open. 2019;9(2):e024010. https://doi.org/10.1136/bmjop en-2018-024010

28. Ishihara M, Harel D, Levis B, et al. Shortening self-report mental health symptom measures through optimal test assembly methods: development and validation of the Patient Health Questionnaire-Depression-4 Depress Anxiety. 2019;36(1):82-92. https://doi.org/10.1002/da.22841

29. Levis AW, Harel D, Kwakkenbos L, et al. Using optimal test assembly methods for shortening patient-reported outcome measures: development and validation of the Cochin Hand Function Scale-6: a scleroderma patient-centered intervention network cohort study. Arthritis Care Res (Hoboken). 2016;68(11):1704-1713 https://doi.org/10.1002/acr.22893

30. Harel D, Baron M. Methods for shortening patient-reported outcome measures. Stat Methods Med Res. 2019;28(10-11):29923011. https://doi.org/10.1177/0962280218795187

31. Thombs BD, Benedetti A, Kloda LA, et al. Diagnostic accuracy of the Edinburgh Postnatal Depression Scale (EPDS) for detecting major depression in pregnant and postnatal women: protocol for a systematic review and individual patient data meta-analyses. BMJ Open. 2015;5(10):e009742. https://doi.org/10.1136/bmjopen-2015-009742

32. Thombs B, Arthurs E, El-Baalbaki G, Meijer A, Ziegelstein R, Steele R. Risk of bias from the inclusion of already diagnosed or treated patients in diagnostic accuracy studies of depression screening tools. Am J Epidemiol. 2011;173:S324.

33. Sampson M, McGowan J, Lefebvre C, Moher D, Grimshaw J. PRESS: Peer Review of Electronic Search Strategies. Ottawa, ON Canadian Agency for Drugs and Technologies in Health; 2008.

34. First MB. Structured clinical interview for the DSM (SCID). In RL Cautin, SO Lilienfeld (Eds.), The Encyclopedia of Clinical Psychology. 2014;1-6.

35. Levis B, Benedetti A, Riehm KE, et al. Probability of major depression diagnostic classification using semi-structured versus fully structured diagnostic interviews. Br J Psychiatry. 2018;212(6):377385. https://doi.org/10.1192/bjp.2018.54

36. Levis B, McMillan D, Sun Y, et al. Comparison of major depression diagnostic classification probability using the SCID, CIDI, and MINI diagnostic interviews among women in pregnancy or postpartum: an individual participant data meta-analysis. International Journal of Methods in Psychiatric Research. 2019;28:ARTN e1803. https://doi.org/10.1002/mpr.1803

37. Wu Y, Levis B, Sun Y, et al. Probability of major depression diagnostic classification based on the SCID, CIDI and MINI diagnostic interviews controlling for Hospital Anxiety and Depression Scale depression subscale scores: an individual participant data meta-analysis of 73 primary studies. J Psychosom Res. 2020;129:109892. https://doi.org/10.1016/j.jpsychores.2019.109892

38. Muthen L, Muthen B. Mplus user's guide, statistical analysis with latent variables. Confirmatory Factor Analysis and Structural Equation Modeling. Los Angeles, CA: Muthen and Muthen; 1998;2012(55):111.

39. McDonald RP, Ho MH. Principles and practice in reporting structural equation analyses. Psychol Methods. 2002;7(1):64-82 https://doi.org/10.1037/1082-989x.7.1.64

40. Chen FN, Curran PJ, Bollen KA, Kirby J, Paxton P. An empirical evaluation of the use of fixed cutoff points in RMSEA test statistic in structural equation models. Sociol Methods Res. 2008;36(4):462-494. https://doi.org/10.1177/0049124108314720

41. Reise SP, Widaman KF, Pugh RH. Confirmatory factor analysis and item response theory: two approaches for exploring 
measurement invariance. Psychol Bull. 1993;114(3):552-566. https://doi.org/10.1037/0033-2909.114.3.552

42. Hu LT, Bentler PM. Cutoff criteria for fit indexes in covariance structure analysis: conventional criteria versus new alternatives. Struct Equ Modeling. 1999;6(1):1-55. https://doi. org/10.1080/10705519909540118

43. Rosseel Y. lavaan: an R package for structural equation modeling. J Stat Soft. 2012;48(2):1-36.

44. Muraki E. A generalized partial credit model - application of an Em algorithm. Appl Psychol Meas. 1992;16(2):159-176. https:// doi.org/10.1177/014662169201600206

45. Rizopoulos D. ltm: an R package for latent variable modeling and item response theory analyses. J Stat Softw. 2006;17(5):1-25.

46. van ver Linden WJ, Boekkooi-Timminga E. A maximin model for IRT-based test design with practical constraints. Psychometrika 1989;54(2):237-247.

47. Harel D. The Effect of Model Misspecification for Polytomous Logistic Adjacent Category Item Response Theory Models. Montreal: McGill University Libraries; 2014.

48. Van der Ark LA. Stochastic ordering of the latent trait by the sum score under various polytomous IRT models. Psychometrika 2005;70(2):283-304. https://doi.org/10.1007/s11336-000-0862-3

49. Cronbach LJ. Coefficient alpha and the internal structure of tests. Psychometrika. 1951;16(3):297-334.

50. Levis B, Negeri Z, Sun Y, Benedetti A, Thombs BD. Accuracy of the Edinburgh Postnatal Depression Scale (EPDS) for screening to detect major depression: systematic review and meta-analysis of individual participant data. BMJ. 2020;371.

51. Youden WJ. Index for rating diagnostic tests. Cancer. 1950;3(1):3235. https://doi.org/10.1002/1097-0142(1950)3:1<32:Aid-Cncr2 820030106>3.0.Co;2-3

52. Bates D, Machler M, Bolker BM, Walker SC. Fitting linear mixed-effects models using lme4. J Stat Softw. 2015;67(1):1-48.

53. Counsell A, Cribbie RA. Equivalence tests for comparing correlation and regression coefficients. Br J Math Stat Psychol. 2015;68(2):292-309. https://doi.org/10.1111/bmsp.12045

54. Liu JP, Ma MC, Wu CY, Tai JY. Tests of equivalence and non-inferiority for diagnostic accuracy based on the paired areas under ROC curves. Stat Med. 2006;25(7):1219-1238. https://doi.org/10.1002/sim.2358

55. Efron B, Tibshirani RJ. An Introduction to the Bootstrap. New York: CRC Press; 1994.

56. Benjamini Y, Hochberg Y. Controlling the false discovery rate - a practical and powerful approach to multiple testing. J R Stat Soc Series B Stat Methodol. 1995;57(1):289-300.

\section{SUPPORTING INFORMATION}

Additional supporting information may be found online in the Supporting Information section.

How to cite this article: Harel D, Levis B, Ishihara $\mathrm{M}$, et al. Shortening the Edinburgh postnatal depression scale using optimal test assembly methods: Development of the EPDS-Dep-5. Acta Psychiatr Scand. 2021;00:1-15. https://doi.org/10.1111/ acps. 13272

\section{APPENDIX 1}

\section{DEPRESSD EPDS Collaboration Group Authors}

Ying Sun, Lady Davis Institute for Medical Research, Jewish General Hospital, Montréal, Québec, Canada; Chen He, Lady Davis Institute for Medical Research, Jewish General Hospital, Montréal, Québec, Canada; Ankur Krishnan, Lady Davis Institute for Medical Research, Jewish General Hospital, Montréal, Québec, Canada; Yin Wu, Lady Davis Institute for Medical Research, Jewish General Hospital, Montréal, Québec, Canada; Parash Mani Bhandari, Lady Davis Institute for Medical Research, Jewish General Hospital, Montréal, Québec, Canada; Dipika Neupane, Lady Davis Institute for Medical Research, Jewish General Hospital, Montréal, Québec, Canada; Zelalem Negeri, Lady Davis Institute for Medical Research, Jewish General Hospital, Montréal, Québec, Canada; Mahrukh Imran, Lady Davis Institute for Medical Research, Jewish General Hospital, Montréal, Québec, Canada; Danielle B. Rice, Lady Davis Institute for Medical Research, Jewish General Hospital, Montréal, Québec, Canada; Marleine Azar, Lady Davis Institute for Medical Research, Jewish General Hospital, Montréal, Québec, Canada; Matthew J. Chiovitti, Lady Davis Institute for Medical Research, Jewish General Hospital, Montréal, Québec, Canada; Nazanin Saadat, Lady Davis Institute for Medical Research, Jewish General Hospital, Montréal, Québec, Canada; Kira E. Riehm, Lady Davis Institute for Medical Research, Jewish General Hospital, Montréal, Québec, Canada; Jill T. Boruff, Schulich Library of Physical Sciences, Life Sciences, and Engineering, McGill University, Montréal, Québec, Canada; Pim Cuijpers, Department of Clinical, Neuro and Developmental Psychology, Amsterdam Public Health research institute, Vrije Universiteit Amsterdam, the Netherlands; Simon Gilbody, Hull York Medical School and the Department of Health Sciences, University of York, Heslington, York, UK; John P. A. Ioannidis, Department of Medicine, Department of Health Research and Policy, Department of Biomedical Data Science, Department of Statistics, Stanford University, Stanford, California, USA; Lorie A. Kloda, Library, Concordia University, Montréal, Québec, Canada; Scott B. Patten, Departments of Community Health Sciences and Psychiatry, University of Calgary, Calgary, Canada; Ian Shrier, Lady Davis Institute for Medical Research, Jewish General Hospital, Montréal, Québec, Canada; Roy C. Ziegelstein, Department of Medicine, Johns Hopkins University School of Medicine, Baltimore, Maryland, USA; Liane Comeau, International Union for Health Promotion and Health Education, École de santé publique de l'Université de Montréal, Montréal, Québec, Canada; Nicholas D. Mitchell, Department of 
Psychiatry, University of Alberta, Edmonton, Alberta, Canada; Marcello Tonelli, Department of Medicine, University of Calgary, Calgary, Alberta, Canada; Jacqueline Barnes, Department of Psychological Sciences, Birkbeck, University of London, UK; Cheryl Tatano Beck, University of Connecticut School of Nursing, Mansfield, Connecticut, USA; Carola Bindt, Department of Child and Adolescent Psychiatry, University Medical Center Hamburg-Eppendorf, Germany; Felipe Pinheiro de Figueiredo, Department of Neurosciences and Behavior, Ribeirão Preto Medical School, Brazil; Gracia Fellmeth, Nuffield Department of Population Health, University of Oxford, Oxford, UK; Barbara Figueiredo, School of Psychology, University of Minho, Portugal; Eric P. Green, Duke Global Health Institute, Durham, North Carolina, USA; Nadine Helle, Department of Child and Adolescent Psychiatry, University Medical Center Hamburg-Eppendorf, Germany; Pirjo A. Kettunen, Department of General Hospital Psychiatry, North Karelia Central Hospital, Joensuu, Finland; Jane Kohlhoff, School of Psychiatry, University of New South Wales, Kensington, Australia; Zoltán Kozinszky, Department of Obstetrics and Gynecology, Danderyd Hospital, Stockholm, Sweden; Angeliki A. Leonardou, First Department of Psychiatry, Women's Mental Health Clinic, Athens University Medical School, Athens, Greece; Sandra Nakić Radoš, Department of Psychology, Catholic University of Croatia, Zagreb, Croatia; Tamsen J. Rochat, MRC/Developmental Pathways to Health Research Unit, Faculty of Health Sciences, University of Witwatersrand, South Africa; Johanne SmithNielsen, Center for Early intervention and Family studies, Department of Psychology, University of Copenhagen, Denmark; Alan Stein, Department of Psychiatry, University of Oxford, Oxford, UK; Robert C. Stewart, Department of Mental Health, College of Medicine, University of Malawi, Malawi; Meri Tadinac, Department of Psychology, Faculty of Humanities and Social Sciences, University of Zagreb, Croatia; S. Darius Tandon, Feinberg School of Medicine, Northwestern University, Chicago, IL, USA; Iva Tendais, School of Psychology, University of Minho, Portugal; Annamária Töreki, Department of Emergency, University of Szeged, Hungary; Thach D. Tran, School of Public Health and Preventive Medicine, Monash University, Melbourne, Australia; Katherine Turner, Epilepsy CenterChild Neuropsychiatry Unit, ASST Santi Paolo Carlo, San Paolo Hospital, Milan, Italy; Mette S. Væver, Centre for Early Intervention and Family Studies, Department of Psychology, University of Copenhagen, Copenhagen, Denmark; Johann M. Vega-Dienstmaier, Facultad de Medicina Alberto Hurtado, Universidad Peruana Cayetano Heredia, Lima, Perú. 